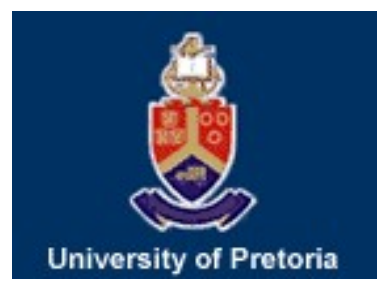

\author{
University of Pretoria \\ Department of Economics Working Paper Series
}

Time-Varying Relationship between Conventional and Unconventional Monetary Policies and Risk Aversion: International Evidence from Time- and Frequency-Domains

Besma Hkiri

University of Jeddah

Juncal Cunado

University of Navarra

Mehmet Balcilar

Eastern Mediterranean University

Rangan Gupta

University of Pretoria

Working Paper: 2019-65

August 2019

Department of Economics

University of Pretoria

0002, Pretoria

South Africa

Tel: +27 124202413 


\title{
Time-Varying Relationship between Conventional and Unconventional Monetary Policies and Risk Aversion: International Evidence from Time- and Frequency-Domains
}

\author{
Besma Hkiri $^{*}$, Juncal Cunado ${ }^{* *}$, Mehmet Balcilar ${ }^{* * *}$ and Rangan Gupta ${ }^{* * * *}$
}

\begin{abstract}
This paper analyzes the time-varying relationship between risk aversion and both conventional and unconventional monetary policy, using Shadow Short Rates, in an international context and at different frequencies during the daily period of 1986-2016, based on a wavelet coherency analysis. Our main results suggest the existence of a dynamic relationship between the two variables depending on timescales and on the periods. Thus, a short-run negative relationship leading from the risk aversion variable to the monetary policy measure is found for most of the period, suggesting that monetary policy reacts more aggressively in period of high risk aversion. Furthermore, during the financial crisis, we find a long-run negative relationship leading from the monetary policy to the risk aversion index, suggesting that a lax monetary policy could lead to financial instability. US monetary policy has also significant effects on the risk aversion rates in the Euro Area, Japan and the UK.
\end{abstract}

Keywords: Risk aversion, Monetary Policy, Wavelet coherency.

JEL classification codes: C49, E44, E52.

${ }^{*}$ University of Jeddah, College of Business, Department of Finance and Economics, Jeddah, Saudi Arabia. Email: bHkiri@uj.edu.sa.

** Corresponding author. University of Navarra, School of Economics, Edificio Amigos, E-31080 Pamplona, Spain. Email: jcunado@,unav.es.

**** Department of Economics, Eastern Mediterranean University, Famagusta, via Mersin 10, Northern Cyprus, Turkey. Email: mehmet@mbalcilar.net.

**** Department of Economics, University of Pretoria, Pretoria, 0002, South Africa. Email: rangan.gupta@up.ac.za. 


\section{Introduction}

A key role of central banks is to conduct monetary policy to achieve price stability (low and stable inflation) and to help manage economic fluctuations (IMF, 2016). With this purpose, and in the wake of the financial crisis, the Federal Reserve eased the monetary policy, lowering the federal funds rate target from 5.25\% in September 2007 to 0.25\% in December 2008 (Mishkin, 2009), and keeping them low to mitigate the effects of the crisis and its aftermath. In this scenario, the effectiveness of the US monetary policy in reducing the effects of the financial crisis has been questioned, since the above policies could have led to an increase in the financial instability through their effects on investors' risk aversion, what is known as the risk-taking channel of the monetary policy. According to this literature (Adrian and Shin, 2009, 2010; Borio and Zhu, 2008, 2012; Jiménez et al., 2014; Bruno and Shin, 2015; Bonfim and Soares, 2018), an environment of low interest rates and low returns of other investments can lead to a reduction in bank portfolio income which might increase banks' willingness to take on more risks while looking for higher returns. Furthermore, low interest rates might affect valuations, incomes and cash flows, and thus, might reduce the banks' risk perception.

On the other hand, the great changes occurred during the last decades in financial markets (technical, regulatory and institutional changes) have transformed investors' willingness to assume and share risks (Rajan, 2006; Guiso et al., 2018), their risk aversion, altering the settings in which monetary policy decisions has to be made, and calling into question whether monetary policy should also seek for financial stability (Gambacorta, 2009; Gambacorta and Marqués-Ibáñez, 2011; Mishkin, 2011). If this were the case, monetary policy could respond to changes in the investors' risk aversion (Gnabo and Moccero, 2015).

In this context, the objective of this paper is to analyse the time-varying relationship between risk aversion and the short-term interest rates in an international context and at different frequencies, using a wavelet coherency analysis. Although the relationship between risk aversion and monetary policy has already been documented in the literature, there are still some issues, such as the persistence of the effect of the monetary policy on risk aversion, the bidirectional relationship between the two variables or the time-varying relationship between them. For instance, Bekaert et al. (2013) use data for the period 1990-2010 and find that lax monetary policies are expected to increase risk aversion in a significant and persistent way, with an effect that lasts for more than two years. However, they do not find a statistically significant effect of risk aversion on monetary policy indicators. On the other hand, Nave and Ruiz (2015) find that a lax monetary policy might increase the risk aversion, although only in the short-run. In contrast, Inekwe (2016) documents bidirectional interdependence between risk aversion and monetary policy, that is, tighter monetary policy induces risk aversion, and at the same time, monetary policy responds to risk aversion. Borio and Zhu (2012) study the link between monetary policy and the perception and pricing of risk and conclude that the interaction between monetary policy and risk-taking behaviour has increased with the financial crisis. Gnabo and Moccero (2015) find that 
monetary policy authorities tend to respond more aggressively in periods of high economic risk. Dajcman (2007), using panel vector autorregression models, finds that the monetary policy affects the risk aversion, concluding that the risk-taking channel in the euro area is operational.

The main contributions of the paper are the following. First, while most papers use VAR-type models to estimate the relationship between these two variables, the wavelet coherency methodology will allow us to determine how the monetary policy-risk aversion relationship evolves through time and frequency. This methodology will help us to understand the lead-lag connection between these two variables across frequencies (short or long term connection) and over time. Second, and in order to overcome the problems of using nominal interest rates in a situation of the Zero Lower Bound, this paper uses the Shadow Short Rate movements as a proxy of the monetary policy, which is not constrained by the Zero Lower Bound limit, and hence is an uniform metric in capturing conventional and unconventional monetary policy simultaneously. Third, the paper extends the analysis to an international context, examining the monetary policy-risk aversion relationship for the US case, the Euro Area, Japan and United Kingdom. To the best of our knowledge, this is the first paper that analyzes the relationship between the Shadow Short Rate and the risk aversion index in an international context using a wavelet coherency analysis. Note that as far as the US is concerned we use daily data over the period of $30^{\text {th }}$ May, 1986 to $30^{\text {th }}$ December, 2016, while for the Euro Area, Japan and the United Kingdom, we rely again on daily data, but cover $3^{\text {rd }}$ January, 1995 to $30^{\text {th }}$ December, 2016. The decision to use highfrequency data for the analysis is motivated by the suggestions of Nakamura and Steinsson (2018a, b), who points out that daily data allows us to identify the monetary policy shocks in a relatively cleaner manner.

Our main results suggest the existence of a dynamic relationship between the two variables depending on timescales and on the periods. Thus, a short-run negative relationship leading from the risk aversion variable to the monetary policy measure is found for most of the period, suggesting that monetary policy reacts more aggressively in periods of high risk aversion. Furthermore, during the financial crisis, we find a long-run negative relationship leading from the monetary policy to the risk aversion index, suggesting that lax monetary policy could lead to financial instability. Our results are in line with those of Borio and Zhu (2012), who argue that recent changes in the financial system may have increased the relevance of the risk taking channel of the monetary policy. Furthermore, our findings also suggest that for most of the analyzed period prior to the financial crisis, monetary policy has reacted to changes in investors' risk aversion. Finally, we also show that US monetary policy has significant effects on the risk aversion rates in the Euro Area, Japan and the UK.

The remainder of the paper is structured as follows. Section 2 describes the methodology. Section 3 presents the data and Section 4 shows the main empirical results. Finally, Section 5 contains some concluding comments and policy implications. 


\section{Methodology Design}

The wavelet tools are suitable to study the extreme co-movements between time series. These approaches are helpful to precisely recognize the short-term and long-term co-movement or, in another word, the cyclical relationship between time series. The wavelet approaches have also demonstrated its ability to explicitly expose and follow the time-scale varying outlines of time series. In our paper, the main objective is to study the time-varying relationship between global risk aversion and the short interest rates in an international context jointly in both frequency and time spaces. A wavelet is a realvalued or a complex valued function $\psi($.$) defined over the real axis. Moreover, it is assumed that the$ wavelet is a square integrable function $\psi(.) \in L^{2}(\mathbb{R})$. In the above equation, $\frac{1}{\sqrt{s}}$ is the normalization factor, ensuring that the unit variance of the wavelet satisfies $\left\|\psi_{u, s}\right\|^{2}=1$ and $u$ denotes the location parameter, providing the exact position of the wavelet. $s$ is the scale dilatation parameter of the wavelet. It defines how the wavelet is stretched or dilated. In this regard, a higher scale implies a more stretched wavelet, which is appropriate for detection of lower frequencies. Formally, the Morlet's wavelet is given by $\psi^{M}(t)=\frac{1}{\pi^{1 / 4}} e^{i \omega_{0} t} e^{-t^{2} / 2}$ where $\psi^{M}(t)$ is the wavelet value at non-dimensional time $t$ and $\omega_{0}$ is the central frequency of the wavelet which is equal to 6 .

\subsection{The continuous wavelet transform}

As in Rua and Nunes (2009) and Baruník et al. (2011), the continuous wavelet transform is given by $W_{x}(u, s)=\int_{-\infty}^{\infty} x(t) \frac{1}{\sqrt{s}} \overline{\psi\left(\frac{t-u}{s}\right)} d t$. Specifically, $W_{x}(u, s)$ is obtained by projecting the specific wavelet $\psi($.$) on the selected time series. The main advantage of the wavelet transform is the aptitude to$ decompose and then consequently reconstruct the function $x(t) \in L^{2}(\mathbb{R})$ :

$x(t)=\frac{1}{C_{\psi}} \int_{0}^{\infty}\left[\int_{-\infty}^{\infty} W_{x}(u, s) \psi_{u, s}(t) d u\right] \frac{d s}{s^{2}}, \quad s>0$

One should note that the main feature of the wavelet transform is the energy preservation of the selected time series. This property is employed for the power spectrum analysis which specifies the variance as follows: $\|x\|^{2}=\frac{1}{c_{\psi}} \int_{0}^{\infty}\left[\int_{-\infty}^{\infty}\left|W_{x}(u, s)^{2}\right| d u\right] \frac{d s}{s^{2}}$.

\subsubsection{Wavelet power spectrum}

The wavelet power spectrum can simply be defined as $\left|W_{n}^{x}\right|^{2}$ and this measure assesses the local variance of each variable (Torrence and Compo,1998; Aguiar-Conraria et al. 2008). The statistical significance, based on works of Grinsted et al. (2004) can be assessed relatively to the null hypothesis that the variable under consideration has a significant power spectrum, i.e., the signal is generated by an $A R(0)$ or $A R(1)$ stationary process with mean background power spectrum $\left(P_{f}\right)$. Based on Monte Carlo simulations through computing the white-noise and red-noise wavelet powers, Torrence and Compo (1998) show 
that, at each time $n$ and scale $s$, the corresponding distribution for the local wavelet power spectrum can be written as

$$
D\left(\frac{\left|W_{n}^{X}(s)\right|^{2}}{\sigma_{X}^{2}}<p\right) \Rightarrow \frac{1}{2} P_{f} \chi_{v}^{2}
$$

where $P_{k}$ is the mean of spectrum at the Fourier frequency $f$ that corresponds to the wavelet scale $s$ $(s \approx 1 / f)$, and $v$ takes the values of 1 or 2 for real or complex wavelets, respectively.

\subsubsection{Cross-wavelet power, wavelet coherence, and phase differences}

The cross-wavelet power shows the area in the time-scale space where the time series exhibit high common power. As noted by Aguiar-Conraria et al. (2008), the cross-wavelet power captures the local covariance of two time series in each frequency and shows the quantitative similarities of power between them. It is also interesting to note that low (high) scales are compressed wavelets allowing us to examine rapidly changing details related with high (low) frequencies, respectively. According to Hudgins et al. (1993), for each signal $X$ and $Y$, the individual wavelet spectra are specified as $W_{n}^{X}(s)$ and $W_{n}^{Y}(s)$, respectively. In the time-frequency analysis, the cross-wavelet between two signals is represented by the cross-wavelet spectrum $W_{n}^{X Y}(s)$ which is defined as in Eq. $(3)^{1}$

$$
W_{n}^{X Y}(s)=W_{n}^{X}(s) W_{n}^{Y^{*}}(s)
$$

where $W_{n}^{Y^{*}}(s)$ is the complex conjugate of $W_{n}^{Y}(s)$ and $*$ denotes complex conjugation. The crosswavelet power is therefore given by $\left|W_{n}^{X Y}\right|$ and it measures the local covariance of two variables at each scale. Torrence and Compo (1998) show that the theoretical distribution of the cross-wavelet power of two signals with background power spectra $P_{k}^{X}$ and $P_{k}^{Y}$ acquires the following form:

$$
D\left(\frac{\left|W_{n}^{X}(s) W_{n}^{Y *}(s)\right|}{\sigma_{X} \sigma_{Y}}<p\right)=\frac{Z_{v}(p)}{v} \sqrt{P_{k}^{X} P_{k}^{Y}}(8)
$$

where $\sigma_{X}$ and $\sigma_{Y}$ designate the standard deviations of $x$ and $y$, respectively. $Z_{v}(p)$ is the confidence interval level related to the probability $p$ for a $p d f$ (probability density function), defined by the square root of the product of two $\chi^{2}$ distributions.

On the other hand, the wavelet coherency of two time series $x=\left\{x_{n}\right\}$ and $y=\left\{y_{n}\right\}$ is defined as the localized correlation coefficient between these series in the time-frequency space (Torrence and Compo, 1998). It is thus a very useful tool for detecting time series' co-movements. Following Torrence and Webster (1999), the wavelet coherence is computed as the squared absolute value of the smoothed

${ }^{1}$ See Torrence and Compo (1998) for more details about cross-wavelet spectrum hypothesis and confidence levels. 
cross-wavelet spectra, normalized by the product of the smoothed individual wavelet power spectra of each time series:

$$
R^{2}(u, s)=\frac{\left|S\left(s^{-1} W_{x y}(u, s)\right)\right|^{2}}{S\left(s^{-1}\left|W_{x}(u, s)\right|^{2}\right) S\left(s^{-1}\left|W_{y}(u, s)\right|^{2}\right)}
$$

where $S$ denotes the smoothing parameter. In the no-smoothing case, the wavelet coherence will be equal to one. Additionally, the squared wavelet coherence coefficient satisfies this inequality $0 \leq R^{2}(u, s) \leq 1$. A value close to zero indicates weak correlation, while a value close to one signifies the presence of high correlation.

While the phase of a wave is defined as a fraction of a complete cycle which oscillates around a time-axis, the phase difference is a difference of the phase between two time series. In addition, the phase difference provides ideas about the lateness of the oscillations between two variables as a function of frequency. The phase difference of two time series, noted as $\phi_{x, y}$, characterizes the phase relationships between them. It effectively gives us information about the time series' positions in the pseudo-cycle. The phase difference is given as;

$$
\phi_{x, y}=\tan ^{-1}\left(\frac{\mathfrak{I}\left\{W_{n}^{x y}\right\}}{\mathcal{R}\left\{W_{n}^{x y}\right\}}\right) \text { with } \phi_{x, y} \in[-\pi, \pi] .
$$

For a more detailed understanding of this issue, we will consider two ideal cyclical time series $X$ and $Y$, where both are sine functions with different phases ${ }^{2}$. The interpretation of the phase as a lead or a lag has to be done relative to the phase difference. Based on Eq.6, we can identify the direction of causality between the two time series. Therefore, when the difference phase is given by $\phi_{1} \in[0, \pi / 2]$ , $X$ leads $Y$ by $\phi_{1}$ and when $\phi_{2} \in[\pi / 2, \pi], X$ lags $Y$ by $\phi_{2}$ (or $Y$ leads $X$ by $\phi_{2}$ ) by $\pi-\phi_{2}$. As well, when the phase difference is $\phi_{3} \in[-\pi,-\pi / 2], X$ leads $Y$ by $\phi_{3}$, or in another words, $X$ leads $Y$ in anti-phase relationship by $\phi_{3}-\pi$ and when $\phi_{4} \in[-\pi / 2,0], Y$ leads $X$ in anti-phase relationship by $2 \pi-\phi_{4}$. However, the relationship between the two time series is unclear when the phase difference is equal to $\pi / 2$ or $-\pi / 2$. According to Ho et al. (2010), to better recognize the lead/lag relation between time series, when the phase difference is $\in[\pi / 2,-\pi / 2]$, it is important to transform each phase of each specified band wavelet into a sine function and make the pseudo cycle, making possible to judge the lead/lag relationship of the two time series at specified band.

In addition, the phase difference is comparable to causality in a Granger sense. In order to identify the direction of causality, which is given by the relative lag between the two time series, we use phase shift, which is interpreted as a lead or a lag between the time series. In this sense we can interpret the

\footnotetext{
${ }^{2}$ We refer the reader to Ho et al. (2010) for definition of these technical issues.
} 
phase difference in terms of the arrow's direction. Arrows pointed to the right (left) indicate that variables are in phase (out of phase or anti-phase). If arrows move to the right and up (down), the first variable $X$ is leading (lagging). By contrast, if arrows move to the left and up (down), the variable $X$ is lagging (leading).

\section{Data}

Our analysis involves two variables, i.e., the measure of risk aversion and the monetary policy metric over the daily period of $30^{\text {th }}$ May, 1986 to $30^{\text {th }}$ December, 2016, with the sample period governed by the data availability on risk aversion. For measuring risk aversion, we utilize the risk aversion index of Bekaert et al., (2017), which is available for download from: https://www.nancyxu.net/risk-aversionindex. These authors develop a new measure of time-varying risk aversion that ultimately can be calculated from observable financial information at high (daily) frequencies. This measure relies on a set of six financial instruments, namely, the term spread, credit spread, a detrended dividend yield, realized and risk-neutral equity return variance and realized corporate bond return variance. An important feature of this measure is that it distinguishes time variation in economic uncertainty (the amount of risk) from time variation in risk aversion (the price of risk) and, thus, provides an unbiased representation for time-varying risk aversion based on a utility function in the hyperbolic absolute risk aversion (HARA) class.

As far as the measure of monetary policy is concerned, with policy rates in the Zero Lower Bound (ZLB) range for a prolonged period of time post the financial crisis included in our analysis, practitioners have been put into a very awkward position of not being able to observe the actual stance of monetary policy. This has posed a great challenge to empirical researchers dealing with monetary policy to find alternative quantitative measures that are able to describe monetary policy at the ZLB. One such measure is the Shadow Short Rate (SSR). The SSR is the nominal interest rate that would prevail in the absence of its effective lower bound, with it derived by modelling the term structure of the yield curve.

The main advantage of the SSR is that it is not constrained by the ZLB and thus allows us to combine the data from the ZLB period with the data from the non-ZLB era, along with the fact that this data is available at the daily frequency to match the risk-aversion. The SSR used in this paper is developed by Krippner (2013), based on models of term-structure, at a daily frequency for the four economies of our concern, and is available for download from the website of the Reserve Bank of New Zealand. ${ }^{3}$ The yield curve-based framework developed by Krippner (2013) essentially removes the effect that the option to invest in physical currency (at an interest rate of zero) has on yield curves,

3 The data can be downloaded from the following link: https://www.rbnz.govt.nz/research-andpublications/research-programme/additional-research/measures-of-the-stance-of-united-states-monetary-

policy/comparison-of-international-monetary-policy-measures. 
resulting in a hypothetical "shadow yield curve" that would exist if physical currency were not available. The process allows one to answer the question: "what policy rate would generate the observed yield curve if the policy rate could be taken negative?" The "shadow policy rate" generated in this manner, therefore, provides a measure of the monetary policy stance after the actual policy rate reaches zero. Both variables have been plotted in Figure A1 in the Appendix of the paper. ${ }^{4}$

\section{Empirical Results}

\subsection{Wavelet coherency, phase differences between US risk aversion and Shadow Short Rate}

Figure 1a corresponds to the wavelet coherence between US risk aversion (hereafter, USRA) and shadow short rate (hereafter, SSRUS). For this plot, the degree of coherency is calibrated by colors ranging from blue to red. While the blue color reveals a small coherency between the time series, the red color indicates a strong correlation between these two variables both across frequencies and over time domains. In addition, the wavelet coherency plot reports the lead-lag relationship between the corresponding variables. For this plot, the relationship is revealed by the arrows directions. These arrows allow for describing the pathway of the causality relationship between SSRUS (in our case Variable $Y$ ) and USRA (Variable $X$ ). While right arrows are synonyms of an in-phase relationship between USRA and SSRUS, left arrows signify that the variables are in anti-phase relationship. As we glean at the Fig 1a, we disclose an interesting conclusion. We precisely reveal a dynamic relationship between SSRUS and USRA depending on timescales and on the periods. More especially, a relatively high opposite comovement between the two variables at medium frequencies (64-512) days of period is remarkably perceived. Furthermore, this negative co-movement is localized at the 1992-2002 sample period. A visual overview on this plot reveals that the arrows are pointed towards the left and down, which suggests that USRA and SSRUS are anti-phase, indicating that the USRA is leading or, in other words, for this timescale, the SSRUS is a follower. The reason behind the opposite relationship between USRA and the proxy of the international monetary policy (SSRUS) may be attributed to the impact of the fluctuation of the risk aversion on the monetary policy. An intensification on the risk aversion leads to drops in the monetary policy measures.

Interestingly, it is also found that, by the end of 2007 to the beginning of 2008, the USRA and SSRUS are in anti-phase in the long-run at the frequency band (512-1024), i.e., a negative relationship between the two variables, indicating that SSRUS was leading in a cyclical effect. This result is not surprising as this period is subsequent to the financial turmoil period where a possibly rising in the global

\footnotetext{
${ }^{4}$ As a preliminary analysis, we estimate a quantile-on-quantile regression as outlined in Sim and Zhou (2015), to understand the underlying relationship between the time-dependent phases of the two variables, i.e., the risk aversion and the interest rate. The results have been plotted in Figures A2 and A3 in the Appendix, and, in general, tends to suggest a theory-consistent negative relationship between the two variables across the various quantiles.
} 
risk aversion may have affected the volatility in financial markets which will be transferred to monetary policy.

Over the whole sample period, while small areas of co-variation are dispersed, the lead-lag relationship could not be viewed clearly. Furthermore, the wavelet coherence plot allows us to perceive a global correlation between the two time series in the time-frequency space whereas the global averaged phase difference permits to check whether the two time-series display phase or anti-phase characteristics and especially whether they exhibit a cyclical relationship.

Figure 1. Wavelet and phase plots for US

1(a). Wavelet coherence between risk aversion and interest rate

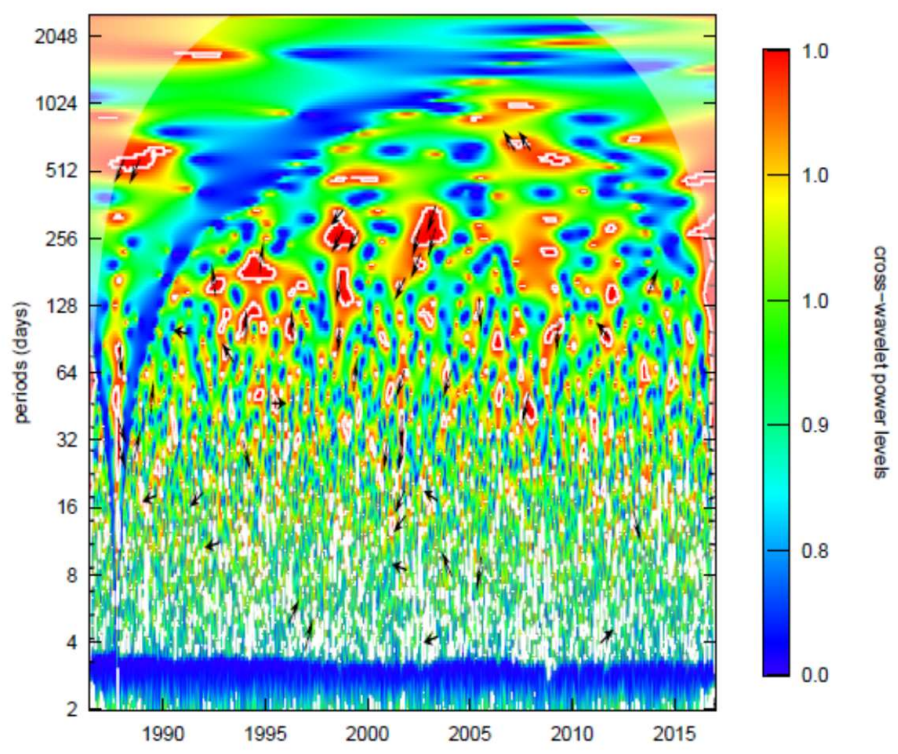




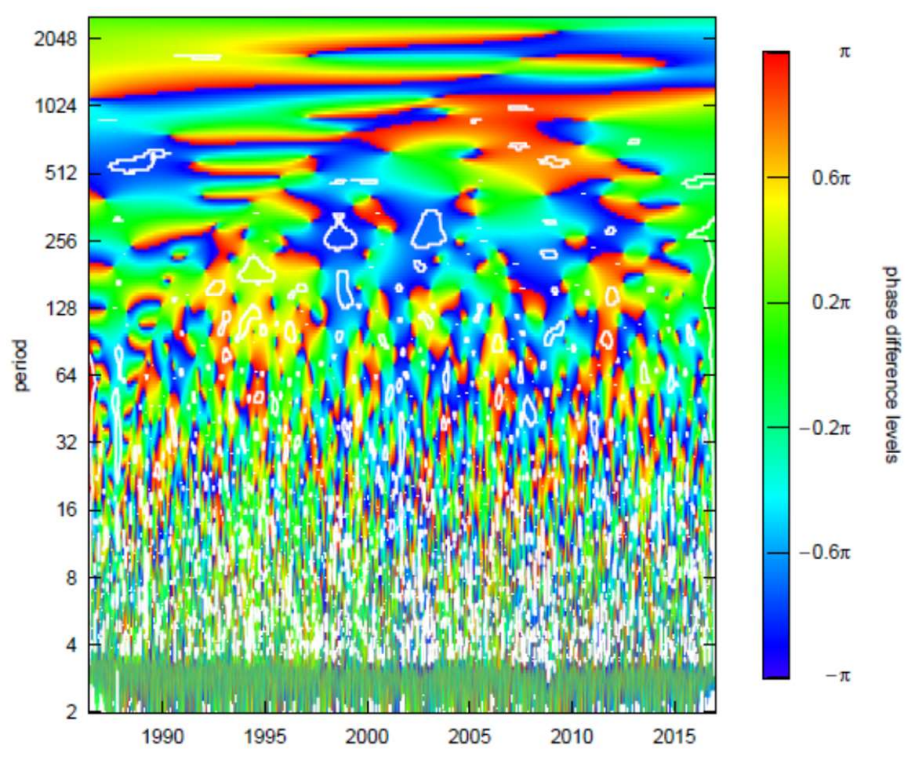

1(c). Phases and time-Lag

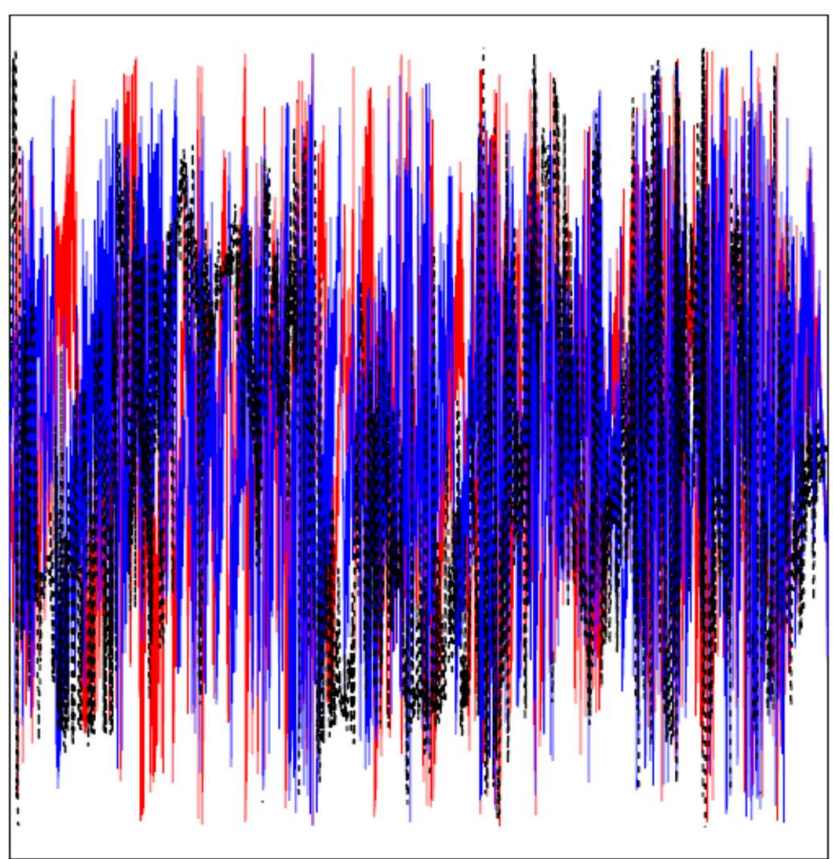

Note: In 1(c), red line is for risk aversion, and blue line for the interest rate, while black-dashed line is for phase difference. The white contour identifies the regions in which the spectrum is significant at the $5 \%$ level against red noise. The cone of influence (COI), indicated by the lighter shade which delimits the high power regions. Time and frequency (daily) are represented on the horizontal and vertical axes, respectively.

The global pattern of averaged phase difference is plotted in the Figure 1b. This graph is essential to enhance our previous results. A looking for this plot proves the co-movement between the SSRUS 
and USRA. More exactly, the two time series strongly co-move at medium and long-run horizons which correspond to the (64-512) and (512-1024) frequency-bands, respectively. As we glean at the wavelet coherence output, these results complement each other. Furthermore, the recent subprime crisis appears to have an impact on the relationship between USRA and SSRUS.

Although the global pattern of averaged difference plot approves a global view of the comovement between the two time-series, the cyclical relationship needs more attention $\mathrm{s}$ it is not straightforward to recognize at average level. For this reason, the pattern of average phase differences will be more attractive to understand the phase/anti-phase relationship between USRA and SSRUS. The aforesaid relationship is plotted in Figure 1c. For this graph, we indicate that the red line symbolizes the US risk aversion proxy (USRA) phase, the blue line is representing the Shadow Short Rate (SSRUS) phase, and the black dotted line shows the phase difference. All these Phases and also Phase-difference 5 which is computed as (Phase. $X$ - Phase. $Y$ ) are shown for different scales and over time. In Figure 1c we detect a remarkable change in the relationship between USRA and SSRUS indicating an alternation in the lead-lag relationship between the two variables. Specifically, in average, the phase difference spins around $\pi / 2$ and 0 for the period of 1995-2000 revealing that the USRA leads the SSRUS in anti-phase relationship. In addition, a notable transformation is shown for the period 2000-2005 when the SSRUS leads the USRA in anti-phase relationship in the $[0,-\pi / 2]$ interval. A visual look to the turmoil period reveals that the USRA and SSRUS move also oppositively. After this episode, the phase plungers over the whole interval $[-\pi / 2, \pi / 2]$ until the end of the sample period where the leading effect of SSRUS on USRA is prounced over the $[0,-\pi / 2]$ interval indicationg that the two time series are in an antiphase relationship. In short, there is a negative relationship between the global risk aversion proxy and shadow short rate for the US case indicating that an increase in the risk aversion leads to a decline in the interest rate. ${ }^{6}$

\subsection{Robustness Analysis: International evidence}

In this sub-section we will discuss the lead-lag relationship between US risk aversion and interest rate for three different advanced regions or countries namely, Euro Area, Japan and United Kingdom.

\footnotetext{
${ }^{5}$ When the phase-difference is converted to an angle in the interval $[-\pi, \pi]$, an absolute value less (larger) than $\pi / 2$ indicates that the two series move in phase (anti-phase).

${ }^{6}$ Given that the risk aversion data is available monthly as well over the period of 1986:06 to 2015:02, in Figure A3 in the Appendix, we analysed the impact of an interest rate (i.e., the shadow short rate) shock on risk aversion, and the feedback from risk aversion on to the interest rate in a vector autoregressive (VAR) model, with growth in industrial production and inflation as additional variables. The data on industrial production and consumer price index is derived from the FRED database of the Federal Reserve Bank of St. Louis. The variables in the VAR (12) is ordered as industrial production growth, inflation, interest rate and risk aversion. As can be seen, from the impulse responses, interest rate and risk aversion moves in opposite direction following a positive shock to either of these two variables. When a robustness test is conducted by replacing risk aversion with the variance risk premium (VRP), as developed by Zhou (2018) (with the data downloadable from: https://sites.google.com/site/haozhouspersonalhomepage/?authuser=1), in the VAR(12) estimated over 1990:01 to 2018:12, the earlier results continue to hold, as seen from Figure A5 in the Appendix.
} 
The main objective of this part is to study how the global risk aversion, which is proxied by the US risk aversion following Demirer et al., (2018), affects the Shadow Short rate for these regions or countries. For the three different cases, the lead-lag relationship between risk aversion and shadow interest rate is reported in Figure 2 (Fig2a to 2c), Figure 3 (Fig 3a to 3c) and Figure 4 (Fig 4a to 4c), respectively. For all these cases, the sample period covered is $3^{\text {rd }}$ January, 1995 to $30^{\text {th }}$ December, 2016, with the start date governed by the availability of data on the shadow short rate and the end date is due to the risk aversion index of the US. The data source for the shadow short rate for the other economies are again derived from the work of Krippner (2013).

\subsubsection{Wavelet coherency, phase difference for Euro Area}

The wavelet coherence between global (US) risk aversion (hereafter, USRA) and shadow short rate for Euro Area case (hereafter, SSREA) is revealed in Fig 2a. From the frequency-time domain sight, the wavelet coherence results display a strong and consistent relationship between the two variables at high scales (low frequencies) indicating that the EURA and SSRE have a long-term relationship. While the highest coherency between USRA and SSREA is localized at the (128-512) frequency-band, the sense of the relationship is not clear. During the period 2000-2004, the arrows are left side downward direction indicating that the USRA and SSREA are in an anti-phase relationship and also having an anticyclic effect where the SSREA leads the USRA by $90^{\circ}$. A same pattern is also shown for the period 2006-2012 indicating that the variables are negatively correlated. Furthermore, a negative relationship is also perceived during 2005-2006 at 512-1024 frequency band which means that the USRA and SSREA have a unidirectional causal relationship with each other in the long-term horizon.

Fig $2 \mathrm{~b}$ is corresponding to the global pattern of averaged difference and reveals that the comovement between USRA and SSREA is localized at low frequencies. Similar to our previous outcome of wavelet coherence, the two time series co-move together in the long term-horizon. To more recognize the direction of this relationship it will be more interesting to examine the pattern of the average phase given in Fig 2c. From this plot, an anti-cyclic relationship is effortlessly shown. From 2003 to 2015, the USRA and SSREA are in anti-phase relationship in the $[0,-\pi / 2]$ interval which signifies that the two variables move appositively which confirming that both variables showing a cyclic effect. 
Figure 2. Wavelet and phase plots for the Euro Area

2(a). Wavelet coherence between risk aversion and interest rate

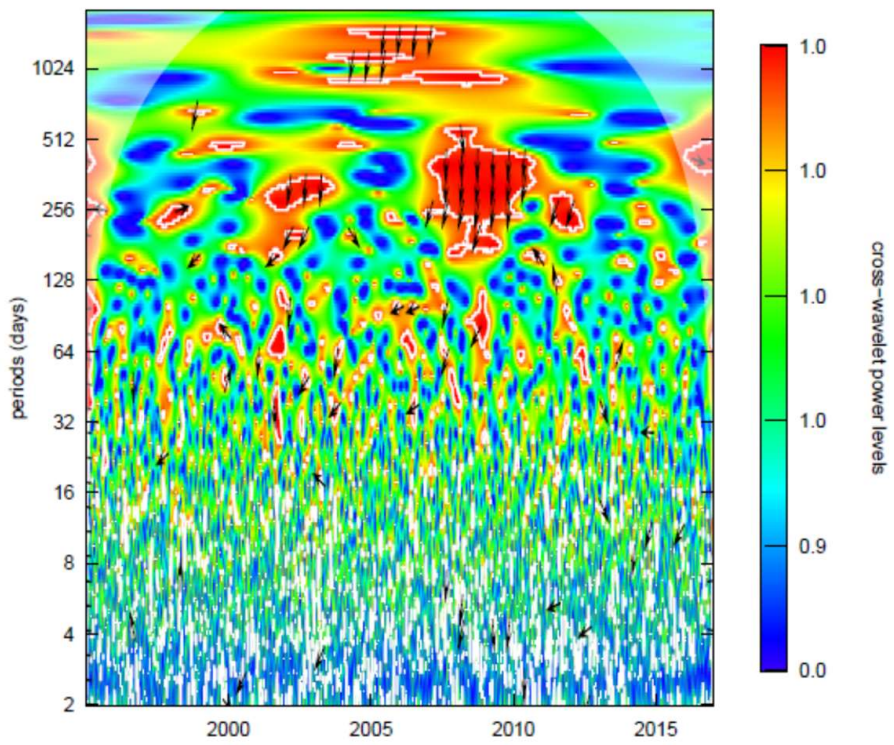

2(b). Global pattern of averaged phase difference

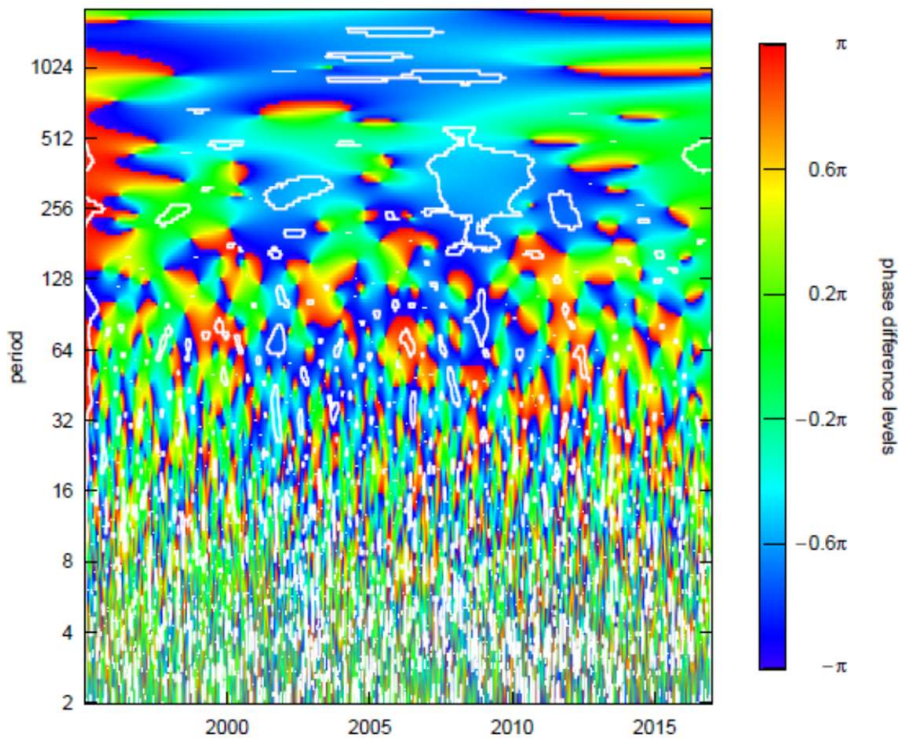




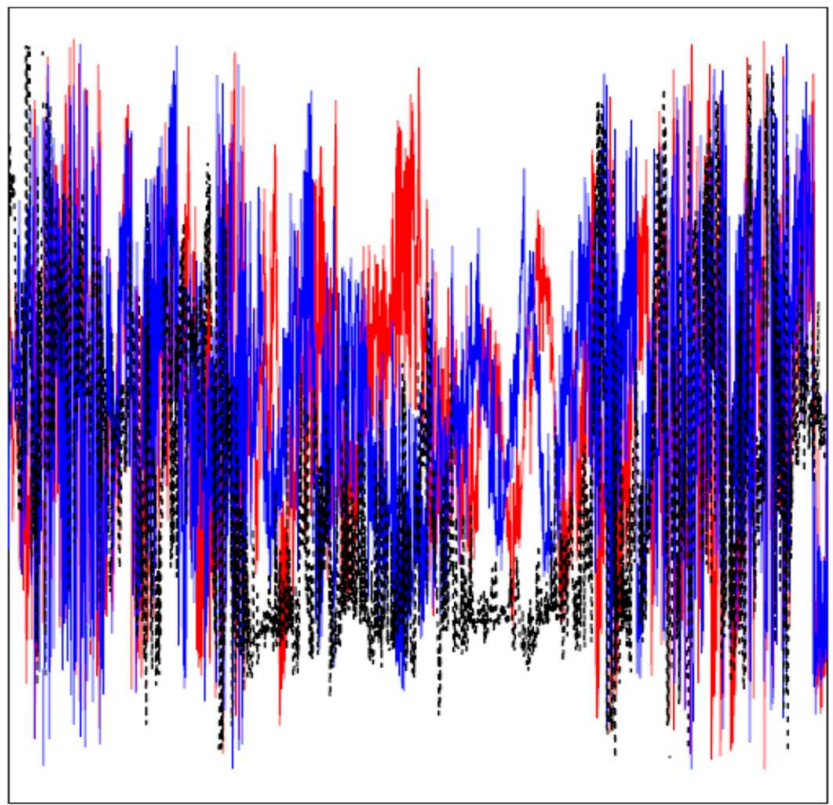

Note: See Notes to Figure 1.

\subsubsection{Wavelet coherency, phase difference for Japan}

For the Japan case reported in Figure 3, the wavelet coherence between global (US) risk aversion (hereafter, USRA) and Shadow Short Rate (hereafter; SSRJ) is revealed in Fig 3a. In general, over the whole sample period and as the arrows are left and downward at medium and high scales (64-256 and 512-1024 periods), the two time series display an anti-phase relationship in which the SSRJ is leading the USRA. The unidirectional causal relationship is running from USRA to SSRJ during 2001 in the long-term horizon (near 1024). There is no interesting relationship in the short-term horizon.

The global pattern of averaged difference plotted in Fig $3 \mathrm{~b}$ confirms our previous findings. The strongest co-movement between USRA and SSRJ is localized at high scales and essentially dispersed over the period 1998-2010. In figure 3c corresponding to the average phase graph, the two variables relationship can be interpreted based on three episodes sorted according the average phase pattern over the sample period. The variables show an anti-phase relationship at the middle of the sample period as the difference phase is included in the $[0,-\pi / 2]$ interval which indicates that they have an anti-cyclic effect and SSRJ leads USRA. Especially, a negative relationship is revealed between the two variables and the USRA is the follower. For the two other episodes, especially at the beginning and the end of the period, noticeable clusters are detected over the $[-\pi / 2, \pi / 2]$ interval. 
Figure 3. Wavelet and phase plots for Japan

3(a). Wavelet coherence between risk aversion and interest rate

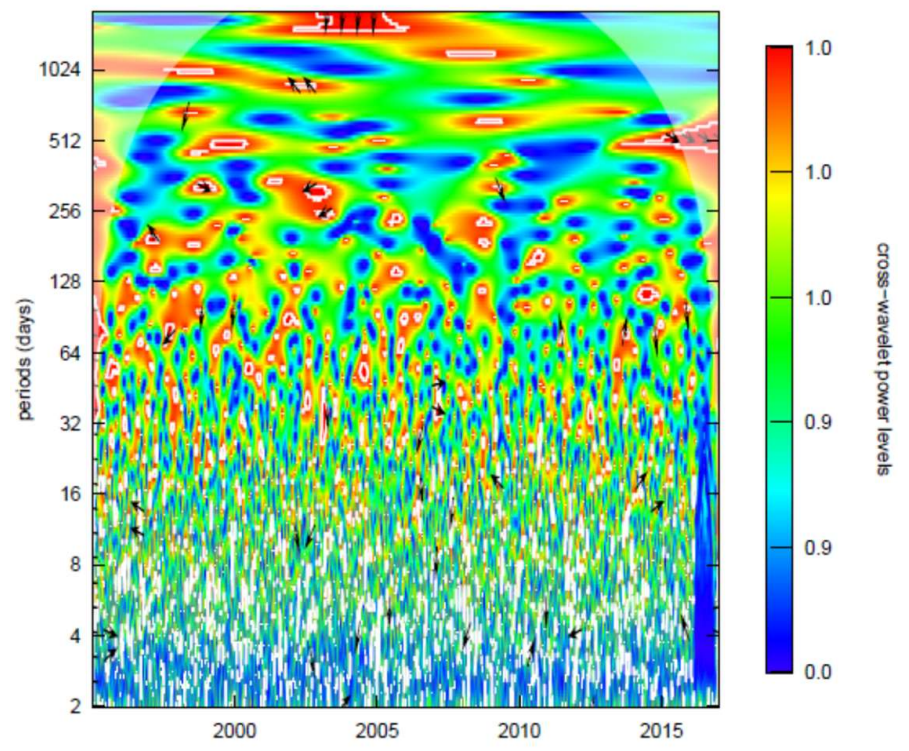

3(b). Global pattern of averaged phase difference

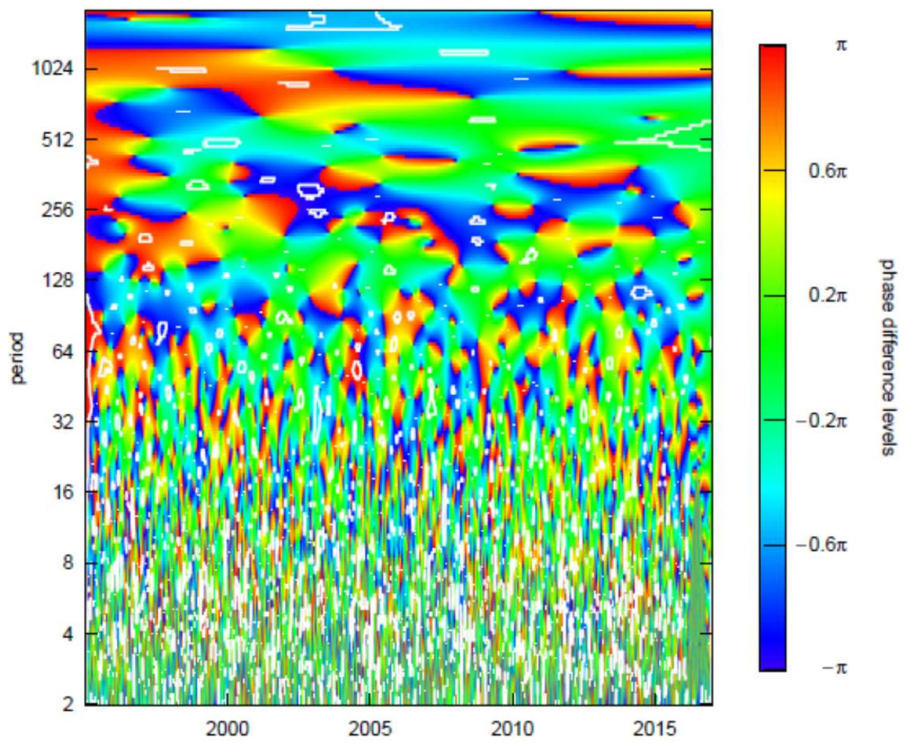




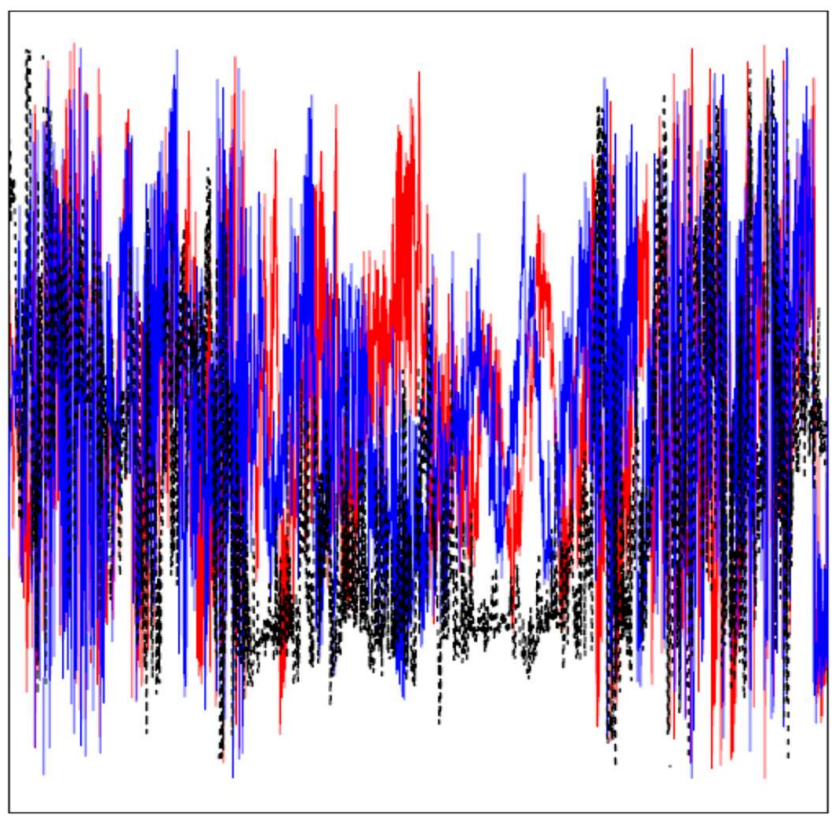

Note: See Notes to Figure 1.

\subsubsection{Wavelet coherency, phase difference for United Kingdom}

In this sub-section, we show the results of wavelet coherence, global averaged difference and average phase differences graphs between the global (US) risk aversion proxy (hereafter, USRA) and Shadow short rate (SSRUK) for the United Kingdom. Figure 4 (Fig 4a to Fig 4c) reported all these plots. When we look for the wavelet coherence between USRA and SSRUK plotted in Fig 4a, we observe comparatively different islands of co-movement between the two variables. Firstly, strong comovements are localized in the medium and long-run horizons which indicates a high dependency between the variables at long and medium horizons. Second, at medium scale or especially at the (64256) frequency-band, two patterns are perceived; from the beginning of the sample period to 2000 the USRA and SSRUK are in phase and having a cyclic effect as the arrows are right side and down explaining that the SSRUK is leading the USRA. Thus, the SSRUK causes adjustment in the aversion risk indicator. From 2001 to 2010 a unidirectional causal relationship is running from SSRUK to USRA as the arrows are left side and down. Similar to the last result, for the long-run horizon (512-1024 scales), the USRA leads the SSRUK in an anti-phase relationship demonstrating an opposite co-movement between the two variables.

Referring to Fig $4 \mathrm{~b}$ corresponding to the global averaged difference, we recognize that the USRA and SSRUK co-move particularly at medium and high horizons over the period 1998 to 2012 which 
confirm the outcomes of the wavelet coherence. The lead-lag relationship between the USRA and SSRUK is revealed in Fig 4c. It is understandable that, at the middle of the sample period, the phase difference is included in the $[0,-\pi / 2]$ interval which signifies that (in average) the SSRUK leads USRA. This finding indicates the negative relationship between the interest rate and the global risk aversion proxy.

Figure 4. Wavelet and phase plots for the UK

4(a). Wavelet coherence between risk aversion and interest rate

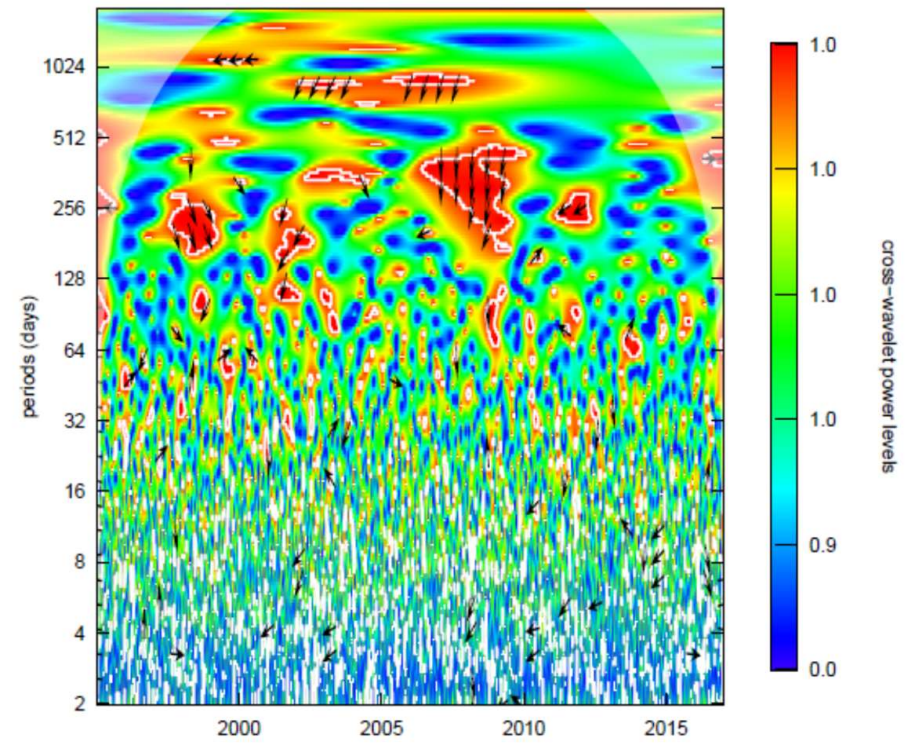

4(b). Global pattern of averaged phase difference

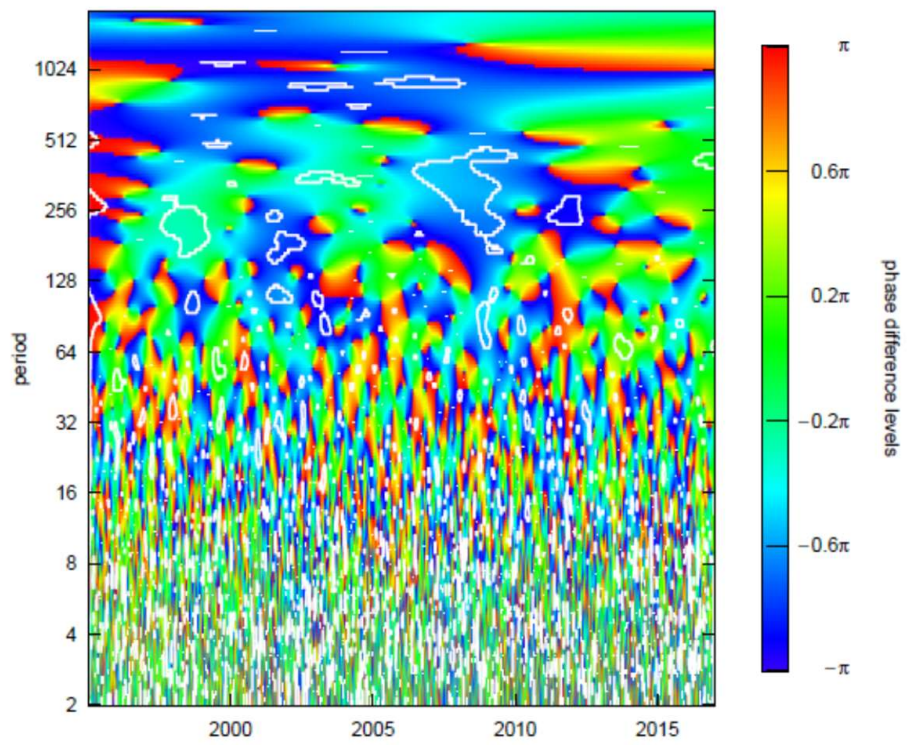




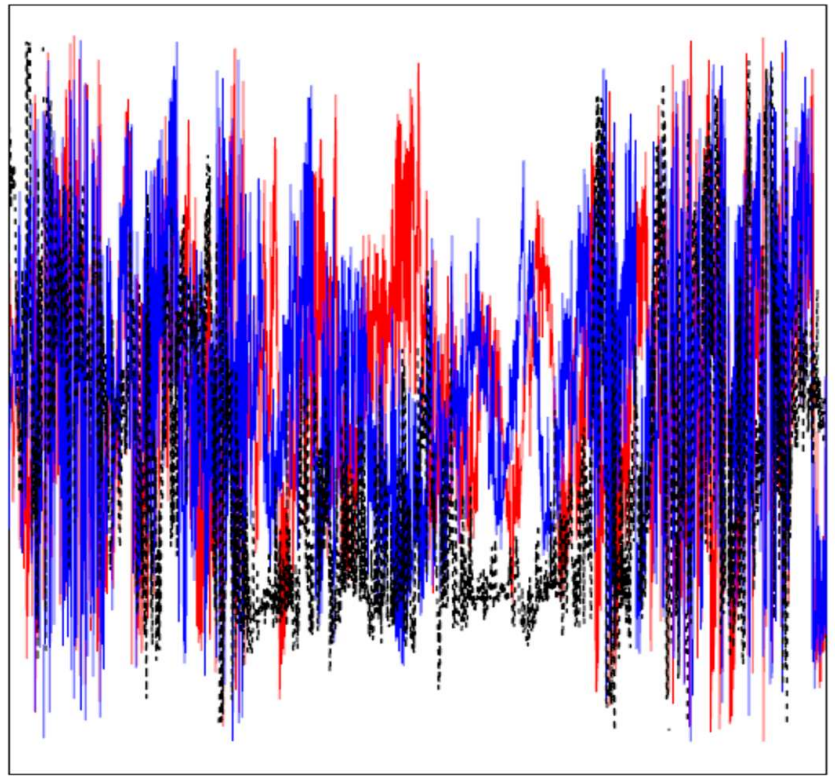

Note: See Notes to Figure 1.

\section{Conclusions}

This paper analyzes the risk aversion-monetary policy relationship in an international context and at different frequencies during the period 1986-2016, using a wavelet coherency analysis. The main results are the following. First, and for the US case, we show that there is an opposite relationship between the risk aversion proxy and Shadow Short Rate (SSR), i.e. a negative leader-follower relationship. For this case, it is perceived that both in the medium and long-runs, the SSR leads the US Risk Aversion (USRA), respectively. The global risk aversion is affected by monetary policy in US indicating thus that the SSR is a crucial factor driving change in the global risk aversion. In addition, from this finding, the USRA and SSR dependency did not depend on scales over time. While a negative relationship is shown during 1995-2000 and 2000-2005 periods, there is an altering of leadership over scales indicating a transformation in the leader-follower relationship. Second, and for all of the other advanced regions or countries, the US risk aversion serving as the global risk aversion proxy, has anti-phase cyclical effect with the Shadow Short Rate. In other words, the Shadow Short Rate has a negative causal effect on the risk aversion indicator. For instance, for the Euro Area case, the pair- wise displays a negative relationship in the long-term run which signify that the Shadow rate causes the risk aversion. Also, during financial crisis, in the long-term horizon, the dependency between the interest rate and the global risk aversion is high and the causality between the two variables is linear (the correlation coefficient is equal to the unity). Generally, same findings are perceived for United Kingdom and Japan cases. At long-term horizons, the Shadow Short Rate is leading the global risk aversion indicator. 
In conclusion, our main results suggest the existence of a dynamic relationship between the two variables depending on timescales and on the periods. Thus, a short-run negative relationship leading from the risk aversion variable to the monetary policy measure is found for most of the period, suggesting that monetary policy reacts more aggressively in period of high risk aversion. Furthermore, during the financial crisis, we find a long-run negative relationship leading from the monetary policy to the risk aversion index, suggesting that a lax monetary policy could lead to financial instability. US monetary policy has also significant effects on the risk aversion rates in the Euro Area, Japan and the UK. As far as monetary policy implications are concerned, our findings suggest that recent changes in the financial system may have increased the relevance of the risk taking channel of the monetary policy. Furthermore, our findings also suggest that for most of the analyzed period prior to the financial crisis, monetary policy has reacted to changes in investors' risk aversion. Moreover, US monetary policies are expected to change risk aversion rates in an international context, as our results for the Euro Area, Japan and the UK suggest.

Given that we are dealing with a variable related to risk, i.e., risk aversion, as part of future research, it would be interesting to extend our analysis to second moments, which is likely to be affected as recently highlighted by Mumtaz and Theodoridis (2019) when dealing uncertainty (which has been shown to have a high positive correlation with risk-aversion by Bekaert et al., (2017)) and the macroeconomy. ${ }^{7}$

\footnotetext{
${ }^{7}$ In fact, preliminary evidence, based on the simultaneous test of causality-in-mean and causality-in-variance using a factor double autoregressive (FDAR) model, as developed by Guo et al., (2014), highlights that the null of simultaneous non-causality is strongly rejected in both directions for the US, Euro Area, Japan and the UK, as reported in Table A1 in the Appendix of the paper.
} 


\section{References}

Adrian, T., \& Shin, H.S., 2009. Money, Liquidity and Monetary Policy. American Economic Review, Papers and Proceedings 99.

Adrian, T., \& Shin, H.S., 2010. Financial Intermediaries and Monetary Economics. In Handbook of Monetary Economics, edited by Benjamin M. Friedman and Michael Woodford. New York, 601-650.

Aguiar-Conraria, L., Azevedo, N., \& Soares, M.J., 2008. Using wavelets to decompose the timefrequency effects of monetary policy. Physica A: Statistical mechanics and its Applications, 387(12), 2863-2878.

Baruník, J., Vácha, L. \& Krištoufek, L., 2011. 'Comovement of Central European stock markets using wavelet coherence: Evidence from high-frequency data. IES Working paper 22/2011, IESFSV. Charles University.

Bekaert, G., Engstrom, E.C., \& Xu, N.R., 2017. The time variation in risk appetite and uncertainty. Columbia Business School Research Paper No. 17-108.

Bekaert, G., Hoerova, M., \& Lo Duca, M., 2013. Risk, uncertainty and monetary policy. Journal of Monetary Economics 60, 771-788.

Bonfim, D., \& Soares, C., 2018. The risk-taking channel of Monetary Policy: Exploring all avenues. Journal of Money, Credit and Banking 50, 1507-1541.

Borio, C., \& Zhu, H., 2012. Capital regulation, risk-taking and monetary policy: a missing link in the transmission mechanism? Journal of Financial Stability 8, 236-251.

Bruno, V., \& Shin, H.S., 2015. Capital flow and the risk-taking channel of monetary policy. Journal of Monetary Economics 71, 119-132.

De Groot, O., 2014. The risk channel of monetary policy. International Journal of Central Banking 10, 115- 160 .

Demirer, R., Omay, T., Yuksel, A., \& Yuksel, A., 2018. Global risk aversion and emerging market return comovements. Economics Letters, 173, 118-121.

Gambacorta, L., 2009. Monetary policy and the risk-taking cannel. BIS Quarterly Review, Bank for International Settings.

Gambacorta, L., \& Marqués-Ibáñez, D., 2011. The bank lending cannel: lessons from the crisis. Economic Policy 26, 135-182.

Gnabo, J.Y., \& Moccero, D.N., 2015. The risk management approach to monetary policy, nonlinearity and aggressiveness: the case of the US Fed. European Central Bank WP 1792.

Grinsted, A., Moore, J. C., \& Jevrejeva, S., 2004. Application of the cross wavelet transform and wavelet coherence to geophysical time series. Nonlinear processes in geophysics, 11(5/6), 561-566.

Guiso, L., Sapienza, P., \& Zingales, L., 2018. Time Varying Risk Aversion. Journal of Financial Economics 128, 403-421. 
Guo, S., Ling, S., and \& Zhu, K. (2014). Factor double autoregressive models with application to simultaneous causality testing. Journal of Statistical Planning and Inference, 148, 82-94.

Ho, S.P., Pan, C., Yeh, C., \& Hsu, Y. (2010). Characteristics of and Relations between Housing Cycles and Economic Fluctuations: A Time-Frequency Analysis. Conference: 2nd ReCapNet Conference, Mannheim, Germany. DOI: 10.13140/2.1.4478.544.

Hudgins, L., Friehe, C.A., \& Mayer, M.E., 1993. Wavelet transforms and atmopsheric turbulence. Physical Review Letters, 71(20), 3279.

Inekwe, N.J., 2016. Financial uncertainty, risk aversion and monetary policy, Empirical Economics 51, 939- 961.

International Monetary Fund, 2016. Monetary Policy and Central Banking, https://www.imf.org/en/About/Factsheets/Sheets/2016/08/01/16/20/Monetary-Policy-and-Central-

Banking.

Jiménez, G., Ongena, S., Peydró, J.L., \& Saurina, J., 2014. Hazardous times for monetary policy: what do twenty-three million bank loans say about the effects of monetary policy on credit risk-taking? Econometrica 82, 463-505.

Krippner, L., 2013. A Tractable Framework for Zero Lower Bound Gaussian Term Structure Models. Discussion Paper, Reserve Bank of New Zealand, 2013/02.

Mishkin, F.S., 2009. Is monetary policy effective during financial crises. American Economic Review 99, 573-577.

Mishkin, F.S., 2011. Monetary Policy Strategy: Lessons from the crisis. National Bureau of Economic Research, NBER 16755.

Mumtaz, H., \& Theodoridis, K. (2019). Dynamic effects of monetary policy shocks on macroeconomic volatility. Journal of Monetary Economics. DOI: https://doi.org/10.1016/j.jmoneco.2019.03.011.

Nakamura, E., \& Steinsson, J (2018a). High Frequency Identification of Monetary NonNeutrality: The Information Effect. Quarterly Journal of Economics, 133, 1283-1330.

Nakamura, E., \& Steinsson, J (2018b). Identification in Macroeconomics. Journal of Economic Perspectives, 32, 59-86.

Nave, J.M., \& Ruiz, J., 2015. Risk aversion and monetary policy in a global context. Journal of Financial Stability 20, 14-35.

Rajan, R., 2006. Has finance made the world riskier? European Financial Management 12, 499-533.

Rua, A., \& Nunes, L.C., 2009. International comovement of stock market returns: A wavelet analysis. Journal of Empirical Finance, 16(4), 632-639.

Sim, N. \& Zhou, A., 2015. Oil prices, US stock return, and the dependence between their quantiles. Journal of Banking and Finance, 55, 1-8. 
Torrence, C., \& Compo, G.P., 1998. A practical guide to wavelet analysis. Bulletin of the American Meteorological society, 79(1), 61-78.

Torrence, C., \& Webster, P.J., 1999. Interdecadal changes in the ENSO-monsoon system. Journal of Climate, 12(8), 2679-2690.

Zhou, H., 2018. Variance Risk Premia, Asset Predictability Puzzles, and Macroeconomic Uncertainty. Annual Review of Financial Economics, 10, 481-497. 


\section{APPENDIX}

Figure A1. Time series plots of interest rate and risk aversion variables
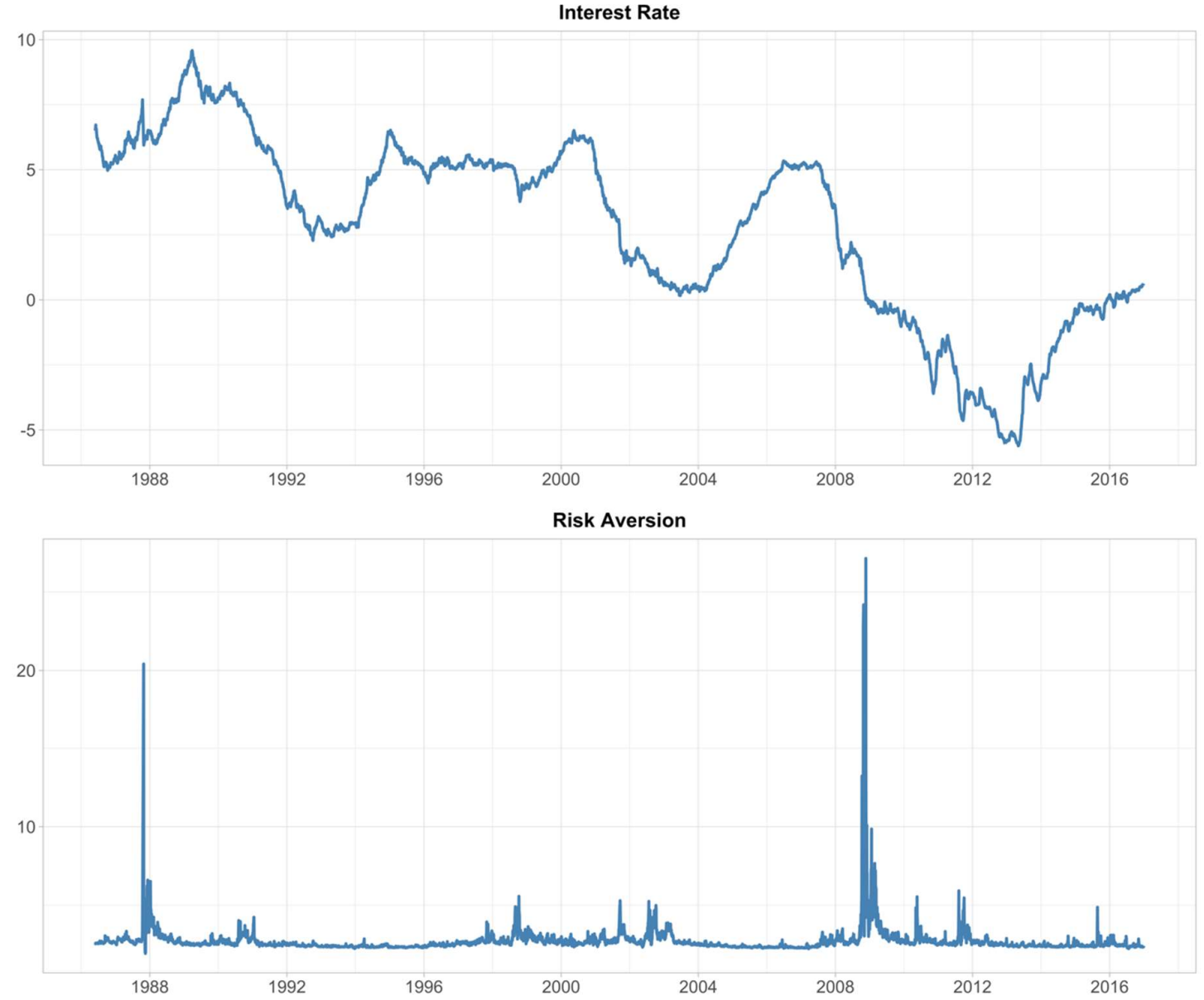

Note: Observations on 19-27 October 1987 for risk aversion variable are not displayed due to large outlier values on these dates, which completely mask the appearance of other observations when included in the plot. 
Figure A2. Quantile-on-quantile slope parameter estimates with interest rate as the dependent variable and risk aversion as the independent variable

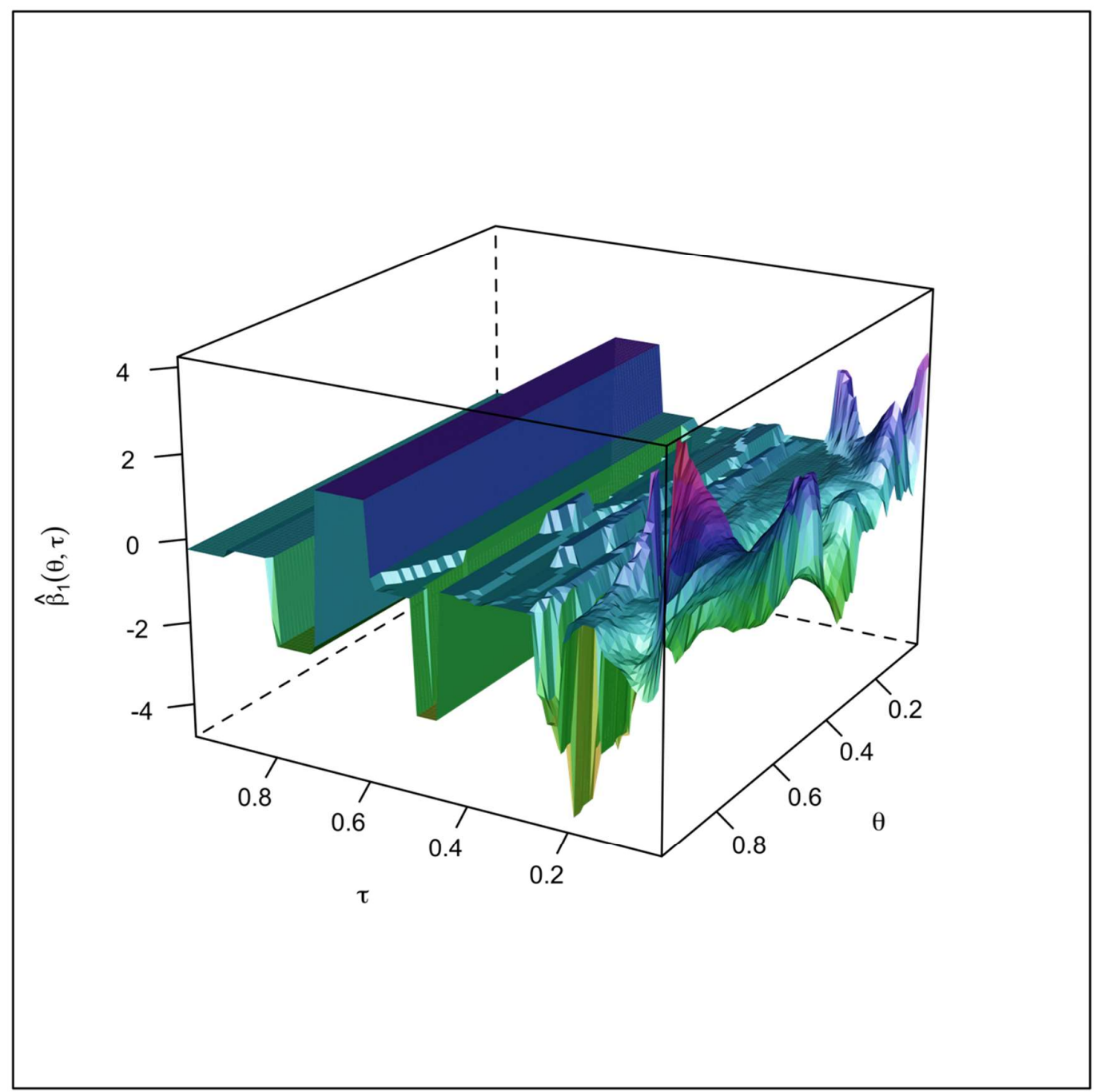

Note: $\theta$ denotes quantiles of interest rate while $\tau$ denotes the quantile of risk aversion. 
Figure A3. Quantile-on-quantile slope parameter estimates with risk aversion as the dependent variable and the interest rate as the independent variable

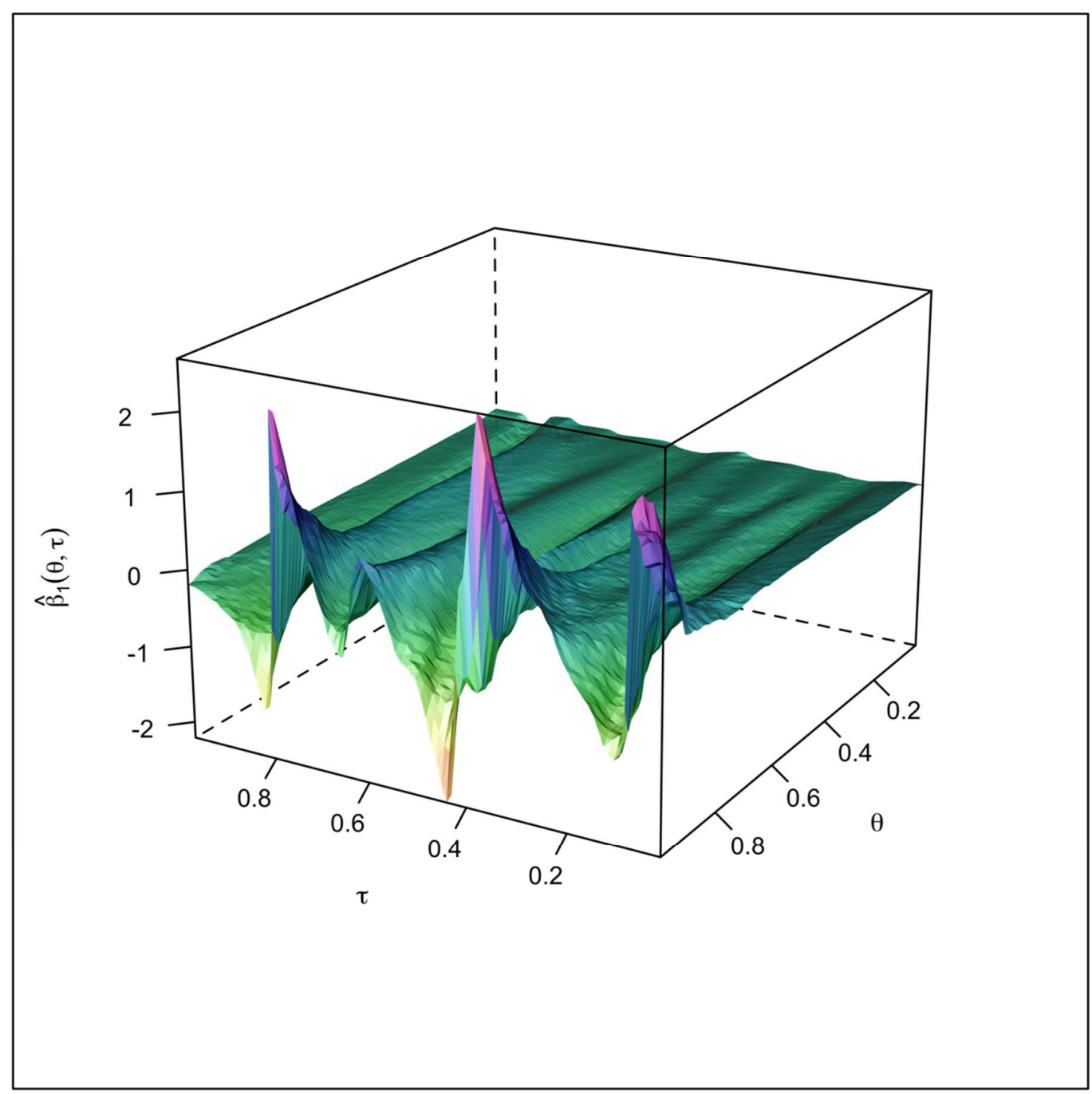

Note: $\theta$ denotes quantiles of risk aversion while $\tau$ denotes the quantile of interest rate. 
Figure A4. Results from Monthly VAR Model with Risk Aversion

$$
\text { Response to Cholesky One S.D. (d.f. adjusted) Innovations } \pm 2 \text { S.E. }
$$

Response of SSRUS to SSRUS

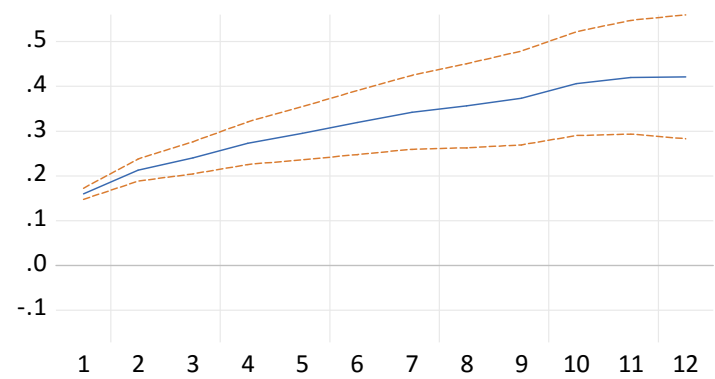

Response of USRA to SSRUS

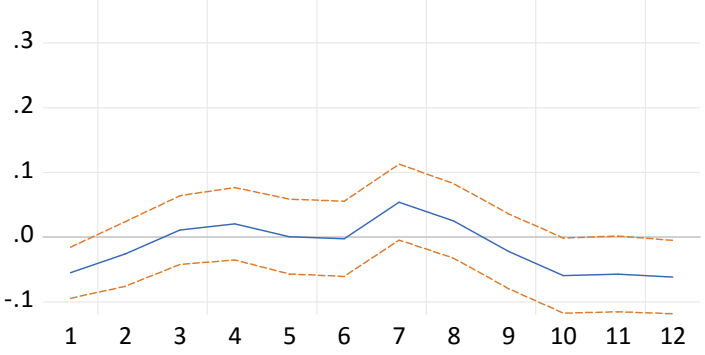

Response of SSRUS to USRA
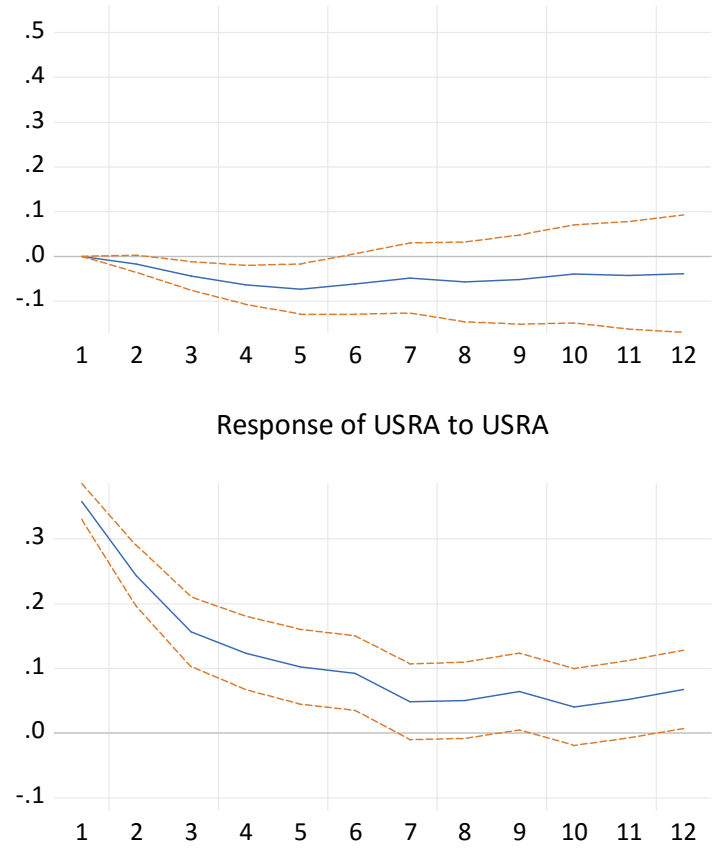

Figure A5. Results from Monthly VAR Model with Variance Risk Premium (VRP)

Response to Cholesky One S.D. (d.f. adjusted) Innovations \pm 2 S.E.

Response of SSRUS to SSRUS
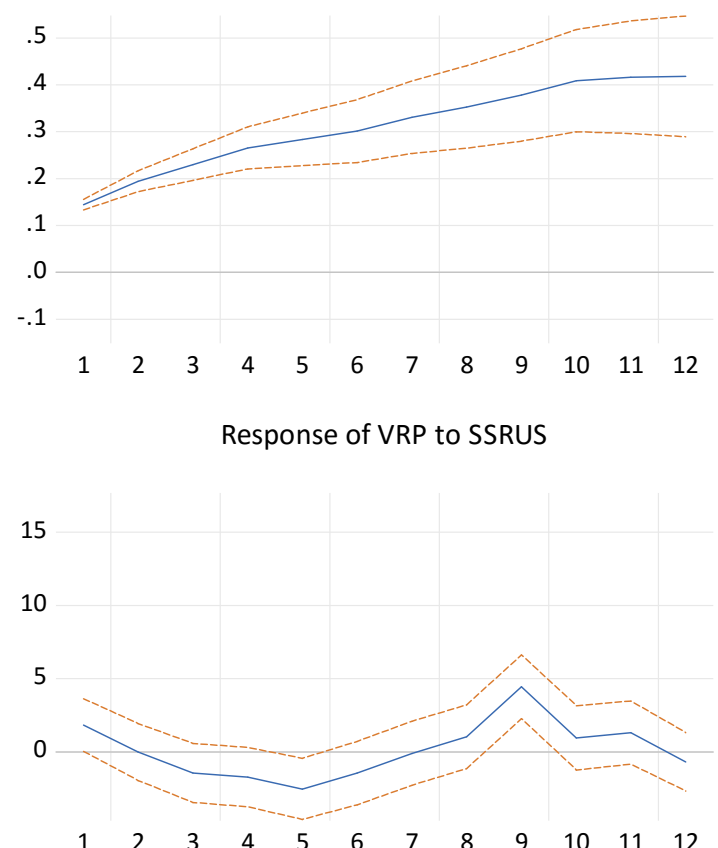

Response of SSRUS to VRP
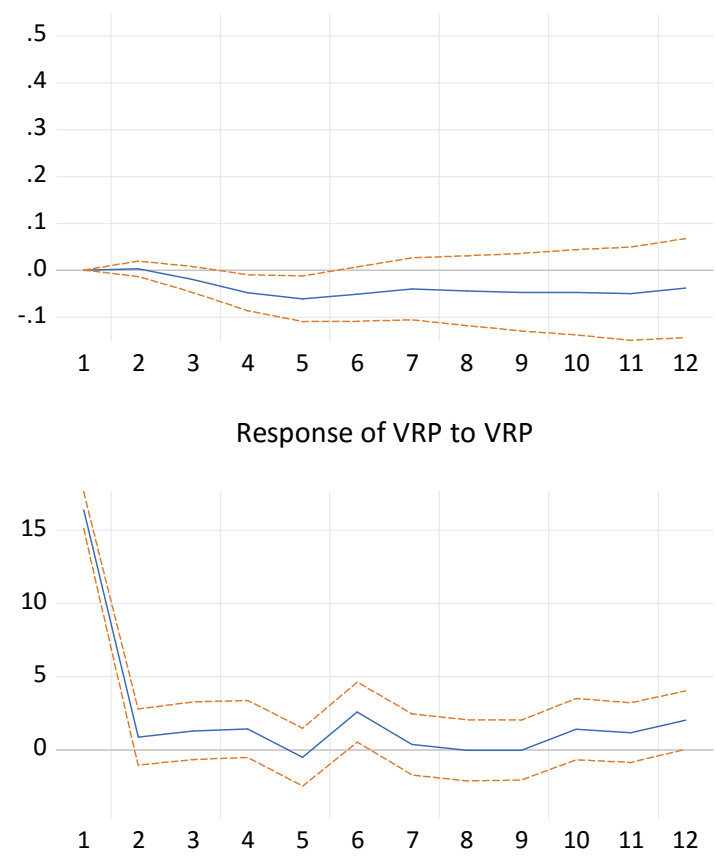
Table A1. Simultaneous test of Causality-in-Mean and Causality-in-Variance

\begin{tabular}{|c|c|c|c|}
\hline Models & $L M$-statistic & $p$-value & Lag Order \\
\hline USRA $\nrightarrow$ SSRUS & 28.6418 & $<0.001$ & 2 \\
\hline SSRUS $\nrightarrow$ USRA & 2149.388 & $<0.001$ & 2 \\
\hline USRA $\nrightarrow$ SSREA & 17.455 & 0.002 & 2 \\
\hline SSREA $\nrightarrow$ USRA & 1151.966 & $<0.001$ & 2 \\
\hline USRA $\nrightarrow$ SSRJ & 21.845 & $<0.001$ & 2 \\
\hline SSRJ $\nrightarrow$ USRA & 806.510 & $<0.001$ & 2 \\
\hline USRA $\nrightarrow$ SSRUK & 49.347 & $<0.001$ & 1 \\
\hline SSRUK $\nrightarrow$ USRA & 1508.161 & $<0.001$ & 2 \\
\hline
\end{tabular}

Note: $\nrightarrow$ indicate does not Granger cause; Lag order is selected by the Schwarz Information Criterion (SIC). 